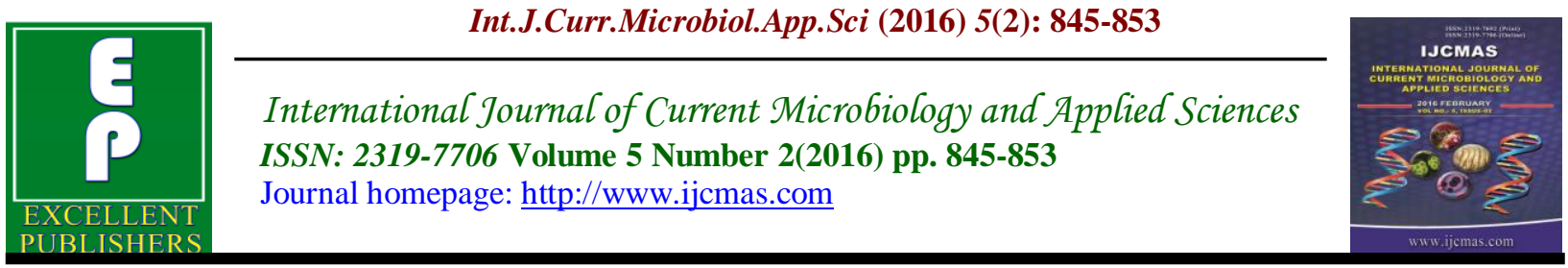

Original Research Article

doi: http://dx.doi.org/10.20546/ijcmas.2016.502.096

\title{
Microbiological Study and Management of Head and Neck Odontogenic Space Infections - A Prospective Study and Review
}

\author{
Ahtesham Ahmad $^{1 *}$, Syed Ahmad ${ }^{1}$, Sheikh Samir ${ }^{1}$, Sandesh Chougule ${ }^{1}$ and Nilofar Ahtesham ${ }^{2}$ \\ ${ }^{1}$ Department of Oral and Maxillofacial Surgery MIDSR Dental college Latur, Maharashtra, India \\ ${ }^{2}$ Global Hospital Latur, Maharashtra, India \\ *Corresponding author
}

\begin{tabular}{|c|c|}
\hline & P \\
\hline & \multirow{3}{*}{$\begin{array}{l}\text { The purpose of this study was to identify microbial flora present in orofacial space } \\
\text { infection of odontogenic origin in order to provide better perspective in the } \\
\text { management of the same. Thirty patients with space infection of odontogenic origin } \\
\text { were selected irrespective of their age and sex. Pus samples were collected and } \\
\text { processed in the microbiology laboratory. The samples were inoculated for aerobic } \\
\text { and anaerobic culturing Demographic profile of the patients showed that male } \\
\text { patients were more commonly involved and most patients fell in to the third and } \\
\text { fourth decade of age groups. Most commonly isolated aerobes were alpha } \\
\text { hemolytic streptococci where as peptococci were the common anaerobes followed } \\
\text { by bacteroids. Clindamycin and amoxicillin-clavulanic acid were the most effective } \\
\text { antibiotics. } 31.8 \% \text { of the anaerobes were resistant to penicillin. In conclusion the } \\
\text { surgical treatment must always be in the foreground for the therapy of abscesses of } \\
\text { odontogenic origin. Decompression through incision and drainage, extraction of the } \\
\text { causative tooth and use of proper antibiotics are the mainstay in the treatment. }\end{array}$} \\
\hline & \\
\hline & \\
\hline
\end{tabular}

\section{Introduction}

Facial space infection has been recognized and described since the time of Galen in the second century. The remains of preColumbian Indians, unearthed in the Midwest, and the remains of people who lived in early Egypt have revealed the bony crypts due to dental abscesses, sinus tracts, and the ravages of osteomyelitis of the jaws ${ }^{1}$.

A fight against microorganism's by the man dates back to ancient civilization. In fact there are references quoting the use of mouldy curds to cure carbuncles by ancient Chinese.
Ancient Indians used chaulmoogra oil to cure leprosy ${ }^{2}$. Despite all these, even after centuries and endless research, mankind has not been successful in eradicating microbial infections in total. The discoveries of sulfonamides by Domg.k and penicillin by Alexander Fleming were encouraging trends towards conquest of the microbial infection ${ }^{2}$. Although penicillin was considered the long awaiting panacea for dental infection the bacteriological spectrum of the oral flora and the understanding of its complexities have undergone rapid evolution since penicillin was introduced ${ }^{1}$. 
Treatment of localized infection was probably the first primitive surgical procedure performed, and it most likely involved the opening of bulging abscesses with sharp stones or pointed sticks. Even today the principle remains the same though the technique has improved. Although therapy has progressed, the scalpel and blade, extraction forceps, and the endodontic reamer remain the keystones of therapy for odontogenic infections, along with the judicious use of antibiotics ${ }^{1}$.

The literature contains many reports of significant morbidity and even mortality from odontogenic space infection, that have resulted in pansinusitis, infective endocarditis, orbital cellulitis, cavernous sinus thrombosis, pharyngeal edema with respiratory embarrassment, and mediastinitis following dental infection that was not effectively controlled. Therefore an understanding of the pathogenic and proper management of oral infections is of critical importance to the dental practitioner.

Before the antibiotic era, most serious odontogenic infections were known to be streptococcal; but the problem of bacterial, resistance to antibiotics soon became obvious in the oral cavity, as elsewhere. The serious epidemic of penicillin resistant staphylococcal infections of the 1950s finally was resolved by the development of the semisynthetic antibiotics which are not metabolized by the penicillinase enzyme of the staphylococci. The widespread use of these drugs has resulted in the current plaque of human infection from enteric (gram negative) and opportunistic organisms, including Vancomycin resistant enterococci and methicillin resistant staphylococci. Mutation and selective genetic pressure have resulted in species that now exhibit resistance to multiple antibiotics, a situation complicated by deoxyribonucleic acid exchange among the species ${ }^{1}$.

This study was done to emphasize the detection of pathogenic microorganisms by microbiological examination and culture of specimen's representative of the infection, importance of early and correct diagnosis of infections, prompt treatment and supportive care.

The main objectives of this study to identify the specific microorganisms causing the infection. To identify the resistant microorganisms causing the infection. And also suggest medical management; thereby provide a better perspective in management of odontogenic infection.

\section{Materials and Methods}

Thirty patients who reported to Department of Oral and Maxillofacial Surgery, MIDSR,Dental College and Hospital,Latur with specific complain of infectious symptoms and diagnosed to have orofacial space infection of odontogenic origin irrespective of their age and sex were selected for the study.

The patients were excluded if they had hypersensitivity to antibiotics. The patients who gave history or those on examination were diagnosed to be immunocompromised (systemic disease or metabolic disorder, congenital defects or primary immunodifeciencies,) were excluded from this study. Pregnant patients were also excluded from this study. Patients who gave history of prior antibiotic medication were excluded from this study.

After taking detailed history each patient was thoroughly examined. The pus was collected from space infection before commencement of antibiotic therapy. 


\section{Specimen Collection}

The extra oral sites were prepared with germicidal soap, alcohol, povidone iodine or a combination of these. Intra oral sites were prepared with chlorexidine. Disposable syringes $(5 \mathrm{ml})$ with disposable needle of 18 gauges were used to aspirate the pus from the abscess. The aspirated syringes with needle were immediately taken to Department of Microbiology, MIMSR Medical College and Hospital, Latur for further investigation.

Pus samples were processed as follows:

1. Smear studies of gram staining

2. Aerobic culture

3. Anaerobic culture

\section{Aerobic Culture}

For aerobic culture the samples were inoculated on Mac-Conkeys agar, blood agar, and nutrient broth. After overnight inoculation the plates were observed for colony formation. The colonies were identified by grams staining and biochemical tests. For gram positive Cocci catalase test, bacitracin sensitivity test, optochin sensitivity test, coagulase test and growth in $6.5 \%$ sodium chloride were used. For gram negative bacilli oxidase test, catalase test, indole test, urease test, citrate test and triple sugar iron were used.

If no growth was observed after the first culture, subcultures from nutrient broth was made on mac-conkey's agar, blood agar and looked for growth after overnight incubation. Growth was identified using appropriate biochemical tests.

\section{Anaerobic Culture}

For anaerobic culture, sample was inoculated into plain blood agar, kanamycin and vancomycin blood agar, bile esculin agar and incubated anaerobically using gas pack, in anaerobic jar for 47 to 72 hours. The plates were observed for colony formation.

The colonies were identified by gram's stain morphology, hemolysis, and sensitivity to antibiotics like penicillin, vancomycin, kanamycin, colistin, growth in bile, indole test, pigmentation, lipase, catalase and sodium polyethanol sulphonate and colonies were tested for aero-tolerance.

If no growth was observed after first culture, subculture was done from Robert cook meet broth on plain blood agar, bile esculin agar and identified as mentioned above.

Antibiotic sensitivity was done by KirbyBauer disk diffusion method for the following drugs.

Penicillin-G, Ampicillin, Amoxycilin, Amoxycillin-clavulanic acid, Cotrimaxazole, Cefotaxime, cephalexin, Gatifloxacillin, Gentamycin, Amikacin, Doxycycline, Metronidazole, Erythromycin, Roxythromycin, Clindamycin.

All patients were started with empirical antibiotics in the form of amoxicillin 500mg every six hours for adults and metronidazole 400mg every eight hours. Severely ill patients were started with cefotaxime IV 1gram every twelve hours and metranidazole IV $500 \mathrm{mg}$ every eight hours. In children doses were adjusted according to patient's age and weight.

Causative teeth were identified by clinical, radiological examination and were immediately extracted. Incision and drainage was carried out under aseptic conditions as follows: All the patients were taken under local or general anesthesia. Incision was given using no 11 blade. Stab 
incision made at most dependent area. Thorough exploration of all the portions of abscess cavity was done by blunt dissection using Lister's sinus forceps. The corrugated rubber drain was placed if required and stabilized with sutures. Drain was kept in place for less than 48 hours.

After culture and sensitivity, depending on the clinical course of the disease appropriate antibiotics were given

\section{Results and Discussion}

\section{Microbiological Flora}

Total of 30 pus samples were studied, 27 were from abscesses of odontogenic origin, one from parotid abscess, one from postoperative infection and one from skin infection. 39 microbes were isolated; no growth was seen in 2 samples (5\%). Out of 38 microbial isolates 37 were bacteria and presence of one fungus (Candida albicans) was identified (Table 1).

The anaerobic Streptococci were seen in 9(22.5\%) samples, Staph. aureus 8 (20\%), Pseudomonas aeruginosa in 8 (20\%), Streptococcus pyogens 4 (10\%), Bacteriodes species 4 (Prevotella melanogenicus 2, Bacteriodes fragilis 1 and Bacteriodes unidentified 1) (10\%), Klebsiella 2(5\%), Chromobacterium violaceum 1(2.5\%), Acenetobacter calcoaceticus 1 (2.5\%), Proteus vulgaris 1 (2.5\%) and Candida albicans 1 (2.5\%) were identified (Table 2, 3).

Pure aerobic organisms were identified in 12 $(40 \%)$ of specimens, pure anaerobes in 6 $(20 \%)$, mixed anaerobes and aerobes in $5(16.7 \%)$, mixed aerobes in $2(6.7 \%)$ and mixed anaerobes were isolated in $1(3.3 \%)$ of specimens.

\section{Antibiotic Susceptibility and Resistance Status}

Anaerobic Streptococci was the most frequently isolated organisms in 9 instances, out of which 3 showed resistance to penicillin. All the 9 organisms were sensitive to amoxicillin, ciprofloxacin, cephotaxime and clindamycin. TABLE 4

Staphylococcus aureus isolated in 8 instances; resistant organisms to penicillin (2), amoxicillin (2), doxycyclin (1), cotrimaxazole (4) and erythromycin (2) were identified. Ciprofloxacin, cephotaxime and gentamycin were effective (100\%) against all these isolates.(TABLE 5)

Out of 8 Pseudomonas aeruginosa isolated all were resistant to penicillin and metronidazole. All isolates were sensitive to gentamycin, cephotaxime and doxycyclin. Streptococcus pyogens isolated in 4 instances, were sensitive to all antimicrobial agents used except metronidazole.

Total of 4 Bacteriodes isolated in this study. Resistant organisms were found 3 to penicillin and one of isolate to erythromycin. Klebsiella, Chromobacterium violaceum, Acinetobacter calcoaceticus and Proteus vulgaris isolated were resistant to penicillin. Ciprofloxacin, cephotaxime and gentamycin were all effective against these organisms.

From the above observation the antimicrobial agents which were most effective against microbiological flora in orofacial abscesses in this study were cephotaxime (97\%), doxycyclin (90\%), ciprofloxacin $(85 \%)$ and amoxicillin $(77 \%)$. $67 \%$ of the total isolates shown resistance to penicillin. 
Table.1 Types of Isolates

\begin{tabular}{|l|l|l|}
\hline Isolates & Frequency & \% \\
\hline a. Aerobic only & 7 & 26 \\
\hline b. Anaerobes only & 8 & 29.5 \\
\hline c. Mixed & 12 & 44.5 \\
\hline Total & 27 & 100 \\
\hline
\end{tabular}

Table.2 Number and Types of Anaerobic Bacteria

\begin{tabular}{|l|c|c|}
\hline Organism & Number of isolates & \% \\
\hline a. Peptostreptococci & 9 & 40.9 \\
\hline b. Bacteroids & 8 & 36.3 \\
\hline c. Prevotella & 3 & 13.6 \\
\hline d. Fusobacterium & 1 & 4.6 \\
\hline e. Porphyromonas & 1 & 4.6 \\
\hline Total & 22 & 100 \\
\hline
\end{tabular}

Table.3 Percentage of Anaerobic Bacteria among Total Number of Isolates

\begin{tabular}{|l|c|}
\hline Organism & \% \\
\hline a. Peptostreptococci & 21.42 \\
\hline b. Bacteroids & 19.04 \\
\hline c. Prevotella & 7.1 \\
\hline d. Fusobacterium & 2.4 \\
\hline e. Porphyromonas & 2.4 \\
\hline Total & 52.4 \\
\hline
\end{tabular}

Table.4 Antibiotic Susceptibility Pattern of Anaerobic Microorganisms

\begin{tabular}{|c|c|c|c|c|c|c|c|c|c|c|c|c|c|c|c|c|c|}
\hline \multirow[t]{2}{*}{ Organism } & \multirow{2}{*}{$\begin{array}{c}\text { Number } \\
\text { of } \\
\text { isolates }\end{array}$} & \multicolumn{2}{|l|}{$\mathbf{P}$} & \multicolumn{2}{|c|}{ Am } & \multicolumn{2}{|c|}{ Amo } & \multicolumn{2}{|c|}{ Acl } & \multicolumn{2}{|c|}{ Co } & \multicolumn{2}{|c|}{ Ce } & \multicolumn{2}{|c|}{$\mathbf{C p}$} & \multicolumn{2}{|c|}{ Ga } \\
\hline & & $\mathbf{S}$ & $\mathbf{R}$ & $\mathbf{S}$ & $\mathbf{R}$ & $\mathbf{S}$ & $\mathbf{R}$ & $\mathbf{S}$ & $\mathbf{R}$ & $\mathbf{S}$ & $\mathbf{R}$ & $\mathbf{S}$ & $\mathbf{R}$ & $\mathbf{S}$ & $\mathbf{R}$ & $\mathbf{S}$ & $\mathbf{R}$ \\
\hline a. Peptostreptococci & 9 & 5 & 4 & 4 & 5 & 5 & 4 & 9 & 0 & 7 & 2 & 6 & 3 & 7 & 2 & 8 & 1 \\
\hline b. Bacteroids & 8 & 7 & 1 & 8 & 0 & 8 & 0 & 8 & 0 & 7 & 1 & 7 & 1 & 7 & 1 & 7 & 1 \\
\hline c. Prevotella & 3 & 3 & 0 & 3 & 1 & 2 & 1 & 3 & 0 & 3 & 0 & 2 & 1 & 3 & 0 & 1 & 2 \\
\hline d. Fusobacterium & 1 & 1 & 0 & 1 & 0 & 1 & 0 & 1 & 0 & 1 & 0 & 1 & 0 & 1 & 0 & 1 & 0 \\
\hline e. Porphyromonas & 1 & 1 & 1 & 1 & 1 & 1 & 1 & 2 & 0 & 0 & 2 & 1 & 1 & 1 & 1 & 1 & 1 \\
\hline
\end{tabular}


Table.5 Antibiotic Sensitivity Pattern of Aerobic Strains

\begin{tabular}{|l|c|c|c|}
\hline Antibiotic & Sensitive & Resistant & \% \\
\hline Penicillin G & 17 & 3 & 15 \\
\hline Ampicillin & 16 & 4 & 20 \\
\hline Amoxicillin & 16 & 4 & 0 \\
\hline Amoxiclav $^{\circledR}$ & 20 & 0 & 15 \\
\hline Cotrimaxazole $_{\text {Cefotaxime }}$ & 17 & 3 & 10 \\
\hline Cephalexin & 18 & 2 & 10 \\
\hline Gatifloxacin & 18 & 2 & 5 \\
\hline Gentamicin & 19 & 1 & 55 \\
\hline Amikacin & 11 & 9 & 25 \\
\hline Doxycycline & 9 & 11 & 55 \\
\hline Metranidazole & 15 & 5 & 20 \\
\hline Erythromycin & 9 & 11 & 10 \\
\hline Roxithromycin & 16 & 4 & 0 \\
\hline Clindamycin & 18 & 2 & 0 \\
\hline
\end{tabular}

Table.6 Distribution of Patients with Different Age Groups

\begin{tabular}{|c|c|c|c|c|c|c|}
\hline $\begin{array}{l}\text { Age groups } \\
\text { (year) }\end{array}$ & Male & $\boldsymbol{\%}$ & Female & $\boldsymbol{\%}$ & Total & $\boldsymbol{\%}$ \\
\hline $0-20$ & 3 & 10 & 1 & 3.3 & 4 & 13.3 \\
\hline $21-40$ & 14 & 46.6 & 8 & 26.6 & 22 & 73.3 \\
\hline $41-60$ & 3 & 10 & 0 & 0 & 3 & 10 \\
\hline $61-80$ & 1 & 3.3 & 0 & 0 & 1 & 3.3 \\
\hline Total & 21 & 70 & 9 & 30 & 30 & 100 \\
\hline
\end{tabular}

Table.7 Frequencies of Causative Teeth

\begin{tabular}{|l|c|c|c|c|}
\hline Causative teeth & Right & Left & Total & \% \\
\hline a.Upper first molar & 4 & 3 & 7 & 18.9 \\
\hline b.Upper second molar & 0 & 1 & 1 & 2.7 \\
\hline c.Upper canine & 2 & 1 & 3 & 8.1 \\
\hline d.Upper central incisor & 1 & 0 & 1 & 2.7 \\
\hline e.Upper lateral incisor & 1 & 0 & 1 & 2.7 \\
\hline f.Upper premolar & 2 & 0 & 2 & 5.4 \\
\hline g.Upper third molar & 0 & 1 & 1 & 2.7 \\
\hline h.Lower deciduous second molar & 0 & 1 & 1 & 2.7 \\
\hline i.Lower third molar & 5 & 1 & 6 & 16.2 \\
\hline j.Lower first molar & 7 & 5 & 12 & 32.4 \\
\hline k.Lower second molar & 1 & 1 & 2 & 5.4 \\
\hline Total & 23 & 14 & 37 & 100 \\
\hline
\end{tabular}


Table.8 Site Distribution of Orofacial Space Infection

\begin{tabular}{|l|c|c|c|c|}
\hline Space & Left & Right & Total & \% \\
\hline a.Canine & 1 & 3 & 4 & 13.33 \\
\hline b.Canine and buccal & 1 & 0 & 1 & 3.33 \\
\hline c.Buccal & 4 & 7 & 11 & 36.66 \\
\hline d.Buccal and submandibular & 2 & 0 & 2 & 6.66 \\
\hline e.Submandibular & 1 & 2 & 3 & 10 \\
\hline f.billateral submandibular & - & - & 1 & 3.33 \\
\hline $\begin{array}{l}\text { f.Bilateral submandibular and } \\
\text { submental }\end{array}$ & - & - & 1 & 3.33 \\
\hline $\begin{array}{l}\text { g.Buccal submandibular and } \\
\text { submental }\end{array}$ & 1 & 0 & 1 & 3.33 \\
\hline $\begin{array}{l}\text { h.Submandibualr } \\
\text { parapharyngeal }\end{array}$ & - & 1 & 1 & 3.33 \\
\hline $\begin{array}{l}\text { i.Submassetric and } \\
\text { j.Palatal }\end{array}$ & 0 & 3 & 3 & 10 \\
\hline Total & - & 2 & 2 & 6.66 \\
\hline
\end{tabular}

Table.9 Antibiotic Sensitivity Pattern of Anaerobic Strains

\begin{tabular}{|l|c|c|c|}
\hline Antibiotic & Sensitive & Resistant & \% \\
\hline Penicillin G & 15 & 7 & 31.8 \\
\hline Ampicillin & 15 & 7 & 31.8 \\
\hline Amoxicillin & 15 & 7 & 31.8 \\
\hline Amoxiclav $^{\circledR}$ & 22 & 0 & 0 \\
\hline Cotrimaxazole & 18 & 4 & 18.2 \\
\hline Cefotaxime & 16 & 6 & 27.3 \\
\hline Cephalexin & 18 & 4 & 18.2 \\
\hline Gatifloxacin & 19 & 3 & 13.6 \\
\hline Gentamicin & 9 & 13 & 59.1 \\
\hline Amikacin & 10 & 12 & 54.5 \\
\hline
\end{tabular}

The microbial residents of the oral cavity constitute one of the most varied floras in the human body. Each organism has a unique set of conditions that permits the organisms to establish residency and thrive, including receptors for selective adherence, appropriate nutrients and oxygen tension or simply physical protection from unfavorable conditions. For these reasons an understanding of the nature of the oral flora and its dynamics is important in Orofacial infections.

In our study thirty patients with Orofacial odontogenic space infections were considered.

The age group most commonly involved was in the third and fourth decades of life. The mean age group was 30.13 . $^{3}$ TABLE 6

Male patients were more commonly involved than female patients. This finding can be compared to the sex distribution given by Goldberg $\mathrm{MH}^{4}$, Kannangara ${ }^{5}$, and females outnumbered males in the study conducted by Hunt D.E ${ }^{6}$. 
Mandibular first molar was the most common causative tooth followed by maxillary first molar and Mandibular third molar. Our findings were similar with the study done by Storoe. $\mathrm{W}^{52}$. (TABLE 7)

The most commonly involved space was buccal followed by submandibular and canine space. This finding was similar to the findings of Storoe $\mathrm{WA}^{7}$, Labriola $\mathrm{TD}^{8}$. (TABLE 8)

\section{Microorganisms}

Odontogenic infection is due to the interdependent and synergistic metabolism of a variety of microorganisms. The roles played by different microorganisms in the group may be difficult to establish but at least in some cases, it has been demonstrated that individual members of the group produce metabolites that are essential for the growth of other microorganisms in the group, they produce substances that create a favourable $\mathrm{pH}$ in the environment, or they consume oxygen and facilitate anaerobic growth. In this study Gram positive cocci were observed in $65.7 \%$ of the gram stain smear study, negative rods in $25.7 \%$ and positive rods in $8.6 \%$ of the cases. These findings were in accordance with findings of Aderhold L ${ }^{9}$, Konow $\mathrm{LV}^{10}$.

Alpha hemolytic streptococci were found in $65 \%$ among aerobes, a finding similar to that of the study by Labriola $\mathrm{TD}^{8}$, Aderhold $\mathrm{L}^{9}$. Beta hemolytic streptococci were present in $15 \%$ of the aerobes isolated. Streptococci were the most common aerobes isolated. This finding was similar to the study conducted by Aderhold $\mathrm{L}^{9}$. But staphylococci were most commonly isolated as per Kannangara $\mathrm{DW}^{11}$.

Anaerobic bacteria were present in 52\% among the total number of isolates finding similar to that of Kannangara DW ${ }^{11}$. Most common anerobe isolated was peptococci, $21.42 \%$ among total isolates followed by bacteroids $19.04 \%$. Similar to the study conducted by Labriola $\mathrm{TD}^{27}$, Aderhold $\mathrm{L}^{9}$

\section{Antibiotic Sensitivity}

Various literature reports that many species of organisms were resistant to penicillin. However most patients were clinically well by the time penicillin resistance was discovered. There are three likely explanations for this: 1) in vitro resistance does not necessarily imply in vivo resistance, particularly in mixed infections; 2) the source of infection had usually been removed and /or surgical incision and drainage accomplished which even without antibiotics, can effect a cure in many cases; 3) the possibility that an interdependent, synergistic, mixed infections, as long as one bacterial species is sensitive to penicillin, the entire pathogenic complex may be rendered nonpathogenic. In the present study penicillin resistance was seen commonly in anaerobes $(31 \%)$. Gentamicin and amikacin had highest percentage of resistance, a finding similar to studies done by Aderhold ${ }^{9}$ (Table 9).

Aminoglycosides are effective in controlling infections due to aerobic gram negative rods which is quite rarely encountered by oral and maxillofacial surgeon. Staphylococci were most frequently resistant to penicillin's among aerobes. All the organisms were found sensitive to clyndamycin and amoxicillin-clavulanate. Most anaerobes were sensitive to metronidazole. Organisms were sensitive to cephalosporins at a higher degree than to penicillin.

In conclusion the surgical treatment must always be in the foreground for the therapy of abscesses of odontogenic origin. 
Decompression through incision and drainage and extraction of the causative tooth and use of proper antibiotics are the mainstay in the treatment.

\section{References}

1. Topazian RG, Goldberg MH, Hupp JR. Oral and maxillofacial infections. $4^{\text {th }}$ ed. Philadelphia: W.B.Saunders company 2002.

2. Tripathi KD. Essentials of medical pharmacology. $5^{\text {th }}$ ed. Jaypee brothers medical publishers (p) limited: New Delhi 2003

3. Virolainen E, Haapaniemi J, Aitasalo K, Suonpaa J. Deep neck infections. Int J Oral Surg 1979;8:407-11

4. Young EW, Johnson WT, Lundin R, Kuker C. Evaluation of treatment provided patients hospitalized with Orofacial odontogenic infections: A retrospective study. Oral Surg Oral Med Oral Pathol 1985;59:28-33

5. Geiseler PJ, Wheat P, Williams RA, Glineberg R. Isolation of anaerobes in Ludwigs angina. J Oral Surgery 1979;37:60-63
6. Goldberg $\mathrm{MH}$. The changing biologic nature of acute dental infection. JADA 1970;80:1048-51

7. Kuriyama T, Karasawa T, Nakagawa K, Saiki Y, Yamamoto E, Nakamura S. Bacteriologic features and antimicrobial susceptibility in isolates from orofacial odontogenic infections. Oral Surg Oral Med Oral Pathol Oral Radiol Endod 2000;90:600-8

8. Dzyak WR, Zide MF. Diagnosis and treatment of lateral pharyngeal space infections. J Oral Maxillofac Surg 1984;42:243-9

9. Greenberg RN, James RB, Marier RL, Hood WH, Sanders CV, Kent JN. Microbiologic and antibiotic aspects of infections in oral and Maxillofacial region. J Oral Surg 1979;37:873-84

10. Bartlet JG, O'Keefe P. The bacteriology of perimandibular space infections. $\mathbf{J}$ Oral Surgery 1979;37:407-9.

11. Monaldo LJ, Bellome J, Zegaralli DJ, Ragaini VE. Bacteroides infection of the mandible with secondary spread to the neck. J Oral Surg 1974;32:370-2

\section{How to cite this article:}

Ahtesham Ahmad, Syed Ahmad, Sheikh Samir, Sandesh Chougule and Nilofar Ahtesham. 2016. Microbiological Study and Management of Head and Neck Odontogenic Space Infections - A Prospective Study and Review. Int.J.Curr.Microbiol.App.Sci.5(2): 845-853. doi: http://dx.doi.org/10.20546/ijcmas.2016.502.096 